\title{
Numerical tool for Shape Memory Alloys structures simulations including twinning effects
}

\author{
Y. Chemisky ${ }^{1}$, A. Duval $^{2}$, B.Piotrowski ${ }^{2}$, T. Ben Zineb ${ }^{2}$, and E. Patoor ${ }^{1, a}$ \\ 1 LPMM, Metz University, Arts et Metiers ParisTech, CNRS, Ile du Saulcy 57045 Metz, France \\ 2 LEMTA, Nancy University, CNRS, 2 rue Jean Lamour, 54500 Vandoeuvre-les-Nancy, France
}

\begin{abstract}
According to the S3T-RoundRobin effort, this paper presents the main results obtained using the SMA model developed by Chemisky et al. [6], from the calibration to the prediction results. This model is based on thermodynamics of the irrevesible processes. Three internal variables are used to model the macroscopic behavior of SMAs. Parameters identification procedure requires only a limited set of experimental data. Comparison between modeling and experimental results are presented for the four data sets of this RoundRobin. Finite Element Analysis was performed to capture tension-torsion tests. Major discrepancies are related to strain localization effects and R-phase transformation which are not included in the present model.
\end{abstract}

\section{Fundamental aspects of modeling}

The present model is dedicated to the description of the macroscopic response of SMAs, as well as the simulation of the response of SMA-based structures, using Finite Element Analysis (FEA). The considered state variables are the average value of inelastic strains in martensite and twinned martensite volumes and the martensite volume fraction inside a representative volume element (RVE). Elastic, thermal and inelastic strains are related to the total strain using an additive decomposition law. Two inelastic strains are considered. The first one is the macroscopic transformation strain which is defined from the martensite volume fraction $f$ and the mean transformation strain $\bar{\varepsilon}_{i j}^{T}$ in the martensite volume [1]:

$$
E_{i j}^{T}=\frac{V_{M}}{V} \frac{1}{V_{M}} \int_{V_{M}} \varepsilon_{i j}^{T}(r) d V=f \bar{\varepsilon}_{i j}^{T}
$$

$V$ denotes the total volume of the considered representative volume element, and $V_{M}$ is the martensite phase volume.

The second inelastic strain is the accommodation of twins which is expressed only in the formed self-accommodated martensite $f^{F A}$, using the mean accommodation of twins strain $[2][3]:$

$$
E_{i j}^{t w i n}=\frac{V_{M^{F A}}}{V} \frac{1}{V_{M^{F A}}} \int_{V_{M^{F A}}} \varepsilon_{i j}^{t w i n}(r) d V=f^{F A} \bar{\varepsilon}_{i j}^{t w i n}
$$

This decomposition lead to a straightforward definition of the internal variables: the volume fraction of martensite $(f)$, the mean transformation strain $\left(\bar{\varepsilon}^{T}\right)$ and the mean accommodation of twins strain $\left(\bar{\varepsilon}^{t w}\right)$. The formed self-accommodated martensite volume is dependant on the amount of martensite formed and the mean transformation strain history (see [2][3] for details).

\footnotetext{
a e-mail: etienne.patoor@ensam.eu
} 
The Gibbs free energy is written over the RVE, following Leclercq and Lexcellent formulation [4]. A specific mixing energy describing the interactions between the two phases is determined with respect to microstructural and macroscopic observations. Gibbs free energy variation from autenite and two-phased state is given by:

$$
\begin{aligned}
\Delta G= & -\Delta T S^{A}+B\left(T-T_{0}\right) f-\frac{1}{2} \Sigma_{i j} S_{i j k l} \Sigma_{k l}-\Sigma_{i j} \alpha \delta_{i j} \Delta T-\Sigma_{i j} f \bar{\varepsilon}_{i j}^{T} \\
& -\Sigma_{i j} f^{F A} \bar{\varepsilon}_{i j}^{t w}+\frac{1}{2} f H_{\bar{\varepsilon}^{T}} \bar{\varepsilon}_{i j}^{T} \bar{\varepsilon}_{i j}^{T}+\frac{1}{2} H_{f} f^{2}+\frac{1}{2} f^{F A} H_{t w i n} \bar{\varepsilon}_{i j}^{t w} \bar{\varepsilon}_{i j}^{t w}
\end{aligned}
$$

where $S^{A}$ is the entropy of austenite, $T_{0}$, the equilibrium temperature of phase transformation $T$, the current temperature and $S$, the fourth order elastic isotropic compliance tensor. Other parameters, related to phase transformation, reorientation and twins accommodation mechanisms are given in Tab. 1 (see next section).

This model also include several additionnal features. Tension-compression asymmetry is taken into account using path direction history dependancy for the maximum transformation strain. Internal loops are considered by introducing discrete memory points formalism initiated by Wack et al [5]. The dissipation potential is expressed to account for the loop hysteresis. Normal rule and additionnal assumptions for non-activated phenomena lead to evolution equations of internal variables. This allows to compute the thermomechanical behavior for non-proportionnal loadingd, and structure simulations can be performed on FEA package like ABAQUS, through a User MATerial (UMAT) subroutine [6].

\section{Parameter identification}

The present model has sixteen parameters to identify, which can be classified in five groups:

- Thermoelastic constants (Elastic parameters and thermal expansion). These paramters are assumed to be homogeneous in both austenite and martensite phases)

- Thermal/mechanical couplings parameters (transformation temperatures and stress-temperature slopes)

- Interaction parameters (which defines the transformation and orientation pseudo-hardening)

- Maximum transformation strains (in tension and in compression)

- Dissipative parameters (or pseudo-yield criteria parameters)

To identify all these parameters, at least four mechanical uniaxial tests have to be performed at different constant temperatures. Three superelastic paths (with one in tension and compression) above $A_{f}$ temperature are needed in order to identify thermal/mechanical couplings parameters and interaction parameters related to phase transformation. One reorientation test below $M_{f}$ temperature is required to identify the parameters related to martensite behavior. Figure 1 summarize the pseudo-phase diagram generated by plotting transformation start and finish stress state at various loading, for the FWM\#1 thin wire used for S3T Roundrobin modeling session. The list of parameters and the adopted values for the simulations are presented in Tab. 1.

It is important to notice that all the parameters are calibrated using only uniaxial tension datas (isothermal) at $-20,0,20$ and $40^{\circ} \mathrm{C}$. As we know that the uniaxial experiment performed at $-20{ }^{\circ} \mathrm{C}$ is not a full orientation behavior but a coupled orientation/transformation behavior. The orientation parameters, as well as the self-accommodated martensite behavior are then difficult to calibrate, and are only estimated by plotting the phase transformation diagram. The model predictions capabilities will be shown on all the other sets of experiments. The reduced number of experiments for the calibration will certainly reduce the model accuracy, but the purpose in this case is to emphasize on the model prediction capabilities based on a simple calibration method. 


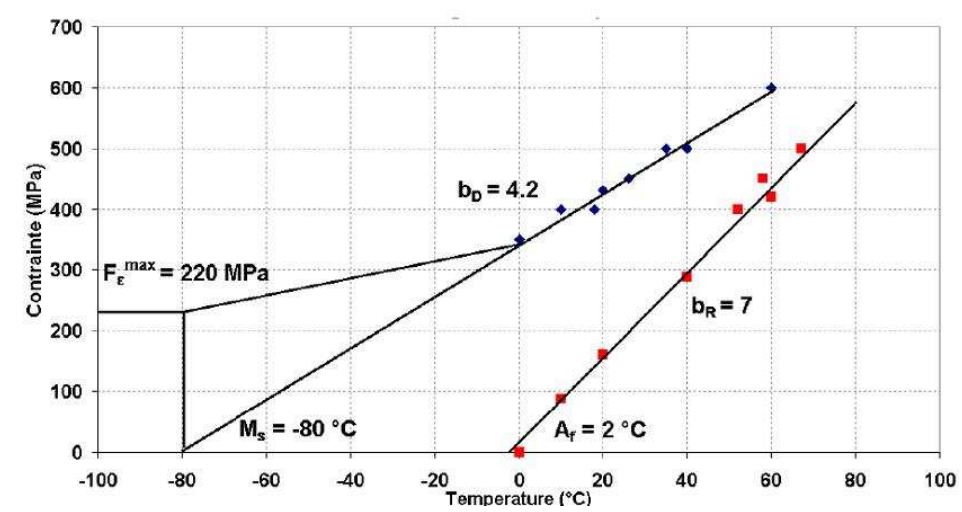

Fig. 1. Pseudo-phase diagram identified for the FWM\#1 thin wire

\begin{tabular}{cccl}
\hline Parameter & Units & Value & Signification \\
\hline$E$ & $\mathrm{MPa}$ & 39500 & Young modulus \\
$\nu$ & - & 0.3 & Poisson coefficient \\
$\alpha$ & ${ }^{\circ} \mathrm{C}^{-1}$ & $8.10^{-6}$ & Thermal expansion coefficient \\
$\varepsilon_{\text {trac }}^{T}$ & - & 0.056 & Saturation value of transformation strain for tension \\
$\varepsilon_{\text {trac }}^{T F}$ & - & 0.053 & Saturation value of transformation strain for tension of self- \\
& & & accommodated formed martensite \\
$\varepsilon_{\text {comp }}^{T}$ & - & $0.044^{*}$ & Saturation value of transformation strain for compression \\
$b_{d t r a c}$ & $\mathrm{MPa} /{ }^{\circ} \mathrm{C}$ & 4.2 & $\begin{array}{l}\text { Direct transformation slope for tension in the pseudo phase } \\
\text { diagram }\end{array}$ \\
$b_{r \text { trac }}$ & $\mathrm{MPa} /{ }^{\circ} \mathrm{C}$ & 7 & Reverse transformation slope for tension in the pseudo phase \\
& & & diagram \\
$M_{s}$ & ${ }^{\circ} \mathrm{C}$ & -80 & Direct transformation start temperature \\
$A_{f}$ & ${ }^{\circ} \mathrm{C}$ & -2 & Reverse transformation finish temperature \\
$r_{f}$ & - & 0.25 & Coefficient for internal loop amplitude \\
$F_{\varepsilon_{T}}^{\text {max }}$ & $\mathrm{MPa}$ & 220 & Yield stress for martensite variants reorientation \\
$H_{f}$ & $\mathrm{MPa}$ & 2 & Pseudo-hardening coefficient for phase transformation \\
$H_{\varepsilon T}$ & $\mathrm{MPa}$ & 1635 & Pseudo-hardening coefficient for reorientation \\
$H_{t w i n}$ & $\mathrm{MPa}$ & $25000^{*}$ & Pseudo-hardening coefficient for twins accommodation \\
$H_{s}$ & $\mathrm{MPa}$ & $68.5^{*}$ & Pseudo-hardening coefficient for martensite stabilisation \\
\hline
\end{tabular}

Table 1. Model parameters adopted for RoundRobin simulation. Parameters with ${ }^{*}$ have been estimated due to a lack of experimental data.

\section{Results of simulations and discussion}

The experimental database is divided into four sets: Uniaxial isothermal complete and partial cycles at various temperatures, full actuation (isobaric) test at various constant stress levels, non proportionnal combined tension/torsion and stress recovery tests at various imposed strains. Numerical simulations will follow the same frame. For each set of experiments, model capabilities are discussed based on comparisons observed between simulations and experiments.

\subsection{Isothermal tension tests}

In Figure 2, simulations and experiments are presented for isothermal tests performed at different temperatures. Several observations are pointed out:

- The first linear part of the uniaxial isothermal tests is well captured when test temperature is in the vicinity of the elastic moduli calibration temperature $\left(-20\right.$ to $\left.20^{\circ} \mathrm{C}\right)$. For higher temperatures, the wire response appears to be stiffer. This can be attributed to 

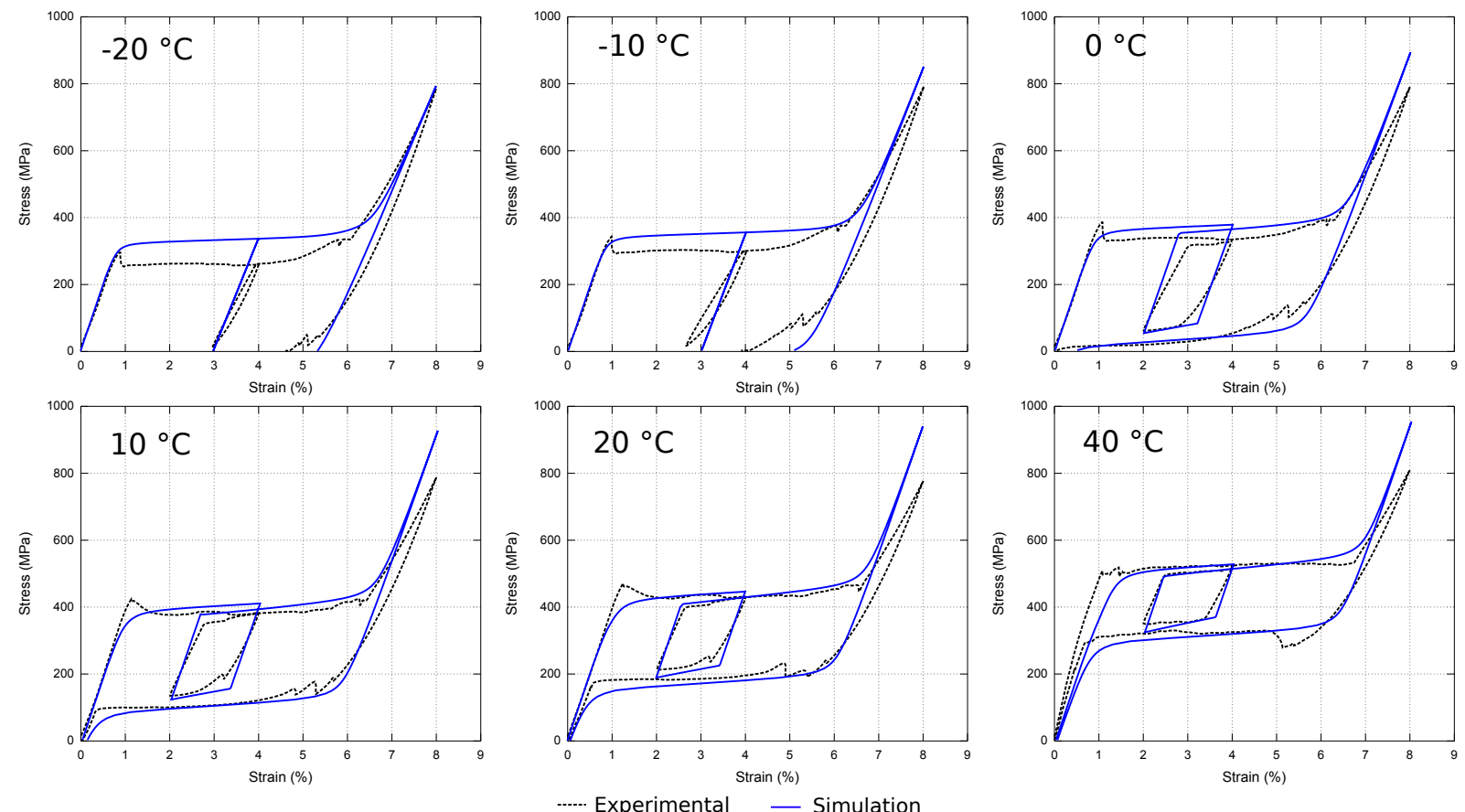

Fig. 2. Isothermal tension tests

the occurence of a R-phase transformation or martensitic transformation in high internal stress state areas, which tends to decrease the apparent moduli for temperatures close to martensitic transformation temperatures.

- The model overpredict the transformation stress at low temperature tests $\left(-20\right.$ and $\left.-10{ }^{\circ} \mathrm{C}\right)$. As the reorientation behavior of the martensite is unknown for this wire, it is not surprising that the combined transformation/reorientation behavior of this model is not accurate. A tension test on a fully martensite sample would certainly improve this estimation.

Beside these particular discrepancies, the model prediction is in very good agreement with uniaxial isothermal experimental results, and partial cycles are well captured.

\subsection{Thermal loadings at constant stress}

For isobaric tests (Figure 3), an experimental weak inelastic strain is obtained for low stress levels. Although there is no experimental evidence, these small actuation strains can be attributed to a R-phase transformation, which is not taken into account in the present model. For higher stress levels (400 to $500 \mathrm{MPa}$ ), the experimental behavior is well described by the model. Transformation temperatures and actuation strains are correctly predicted.

\subsection{Non-proportionnal tension-torsion tests}

Previous simulation were performed using a stand-alone numerical software, by computing the uniform stress response corresponding to a given strain state. In order to take into account the stress gradient versus the wire radius, the tension-torsion tests must be perfomed using a finite element software like ABAQUS. The adopted finite element mesh for a $0.1 \mathrm{~mm}$ portion of the wire is given in Fig. 4. It is made of quadratic hexaedral (20 nodes and 27 integration points) and triangular prismatic (15 nodes and 9 integration nodes) elements.

This set of experiments consists of isothermal loading paths, where an angular displacement is applied at a constant axial stress state (preload). As observed in Figure 5, the model underpredict the resulting torque moment and the yield transformation stress. The ending transformation behavior is also overestimated. Two hypothesis are discussed in order to explain these 

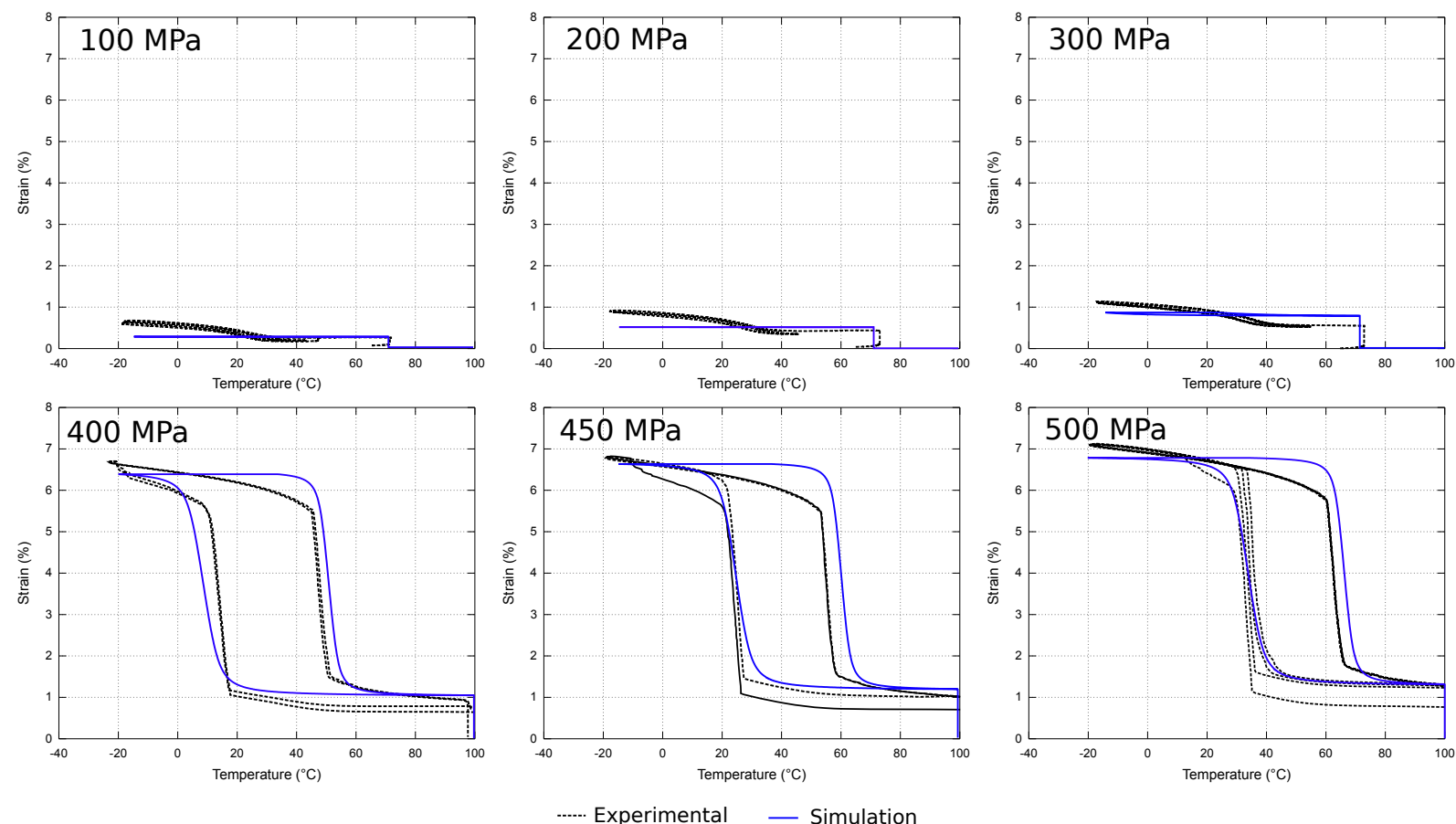

Fig. 3. Thermal loadings at constant stress.

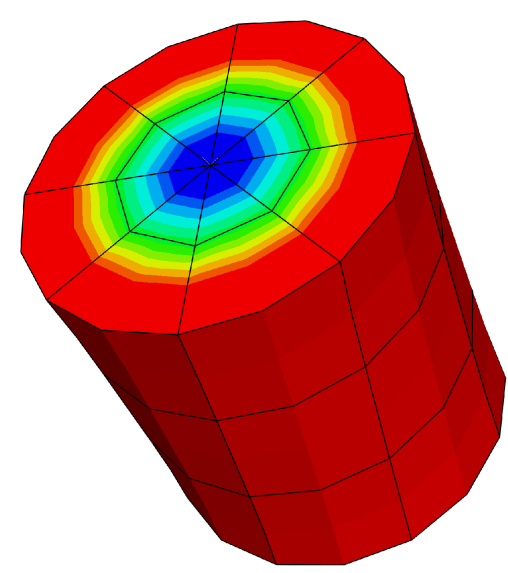

Fig. 4. Finite element mesh for the simulation of tension-torsion loadings. Colors represent the value of martensite volume fraction (blue $=0$, red $=1$ ).

discrepancies. First of all, while all the material paramters are calibrated using tension tests, discrepancies when performing other loading paths are not surprising for NiTi thin wires. Thin wires loaded in tension often exhibit a localization of transformation, which produce more well stress-oriented variants. This results in an important transformation strain in tension. In torsion test, due to the stress gradient through the radius, no localization is expected, then a reduced transformation strain is expected. As the model consider the transformation in an average way, these kind of effect are not captured. This may explain the overestimation of the transformation strain. Another important feature to take into account for this high-cold worked thin wires is the effect of the material texture. Indeed this may strongly influence the yield transformation surfaces and the transformation strains. As the model do not take into account the effect of material texture, this effect is also not captured. beside these discrepncies, the global behavior as the evolution of the axial strain are generally in good agreement with the experiments. 

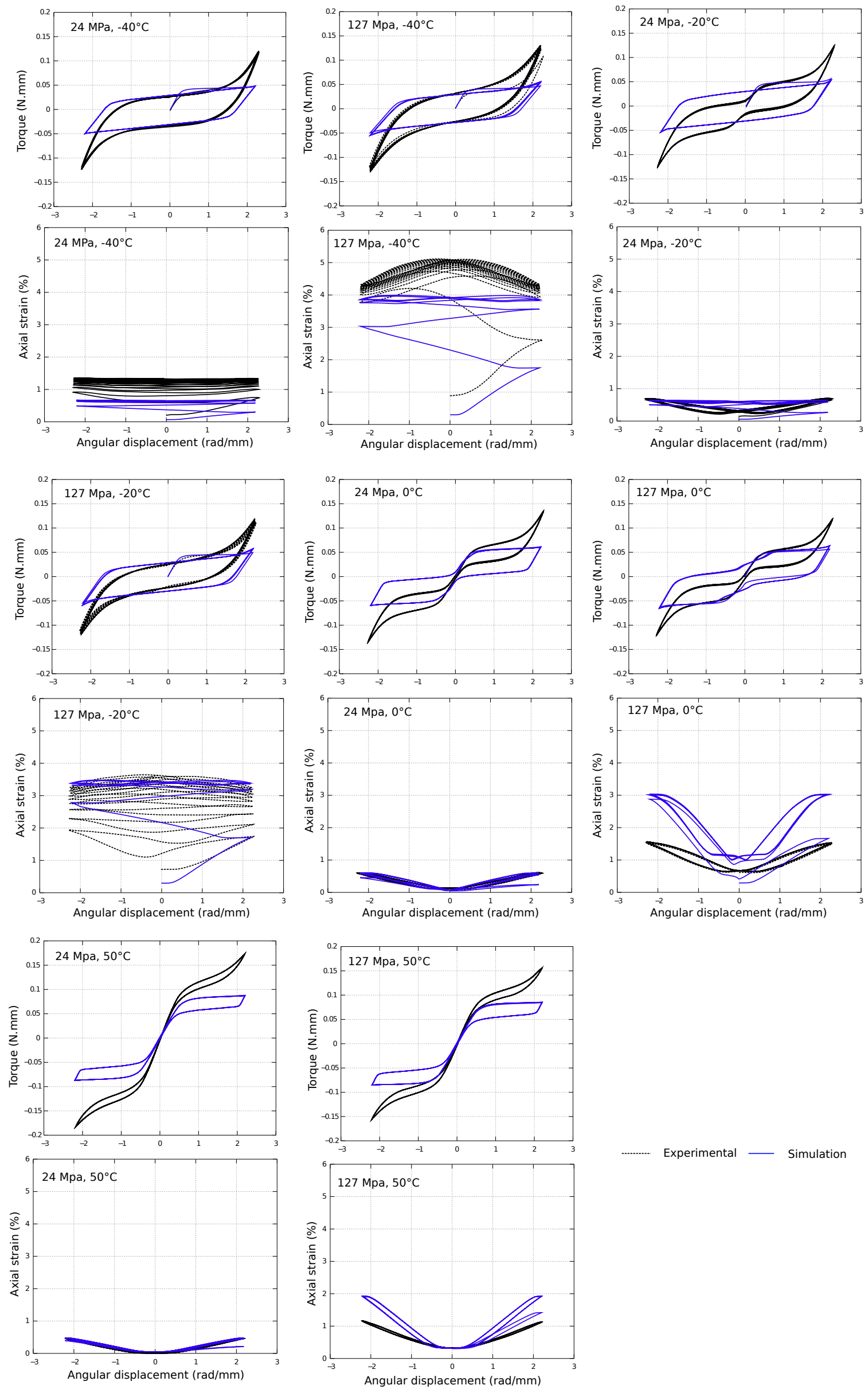

....-.... Experimental _- Simulation

Fig. 5. Isothermal tension-torsion tests. 


\subsection{Stress recovery tests}

The database includes six recovery stress tests, for imposed strain of $2,3.5$ and $6 \%$ on the upper and lower stress plateau. Comparisons between experiments and model predictions are presented in Fig. 6 with $(\Sigma, E)$ and $(\Sigma, T)$ diagrams. The key point to be oberved in these tests is the resulting stress at the end of heating step. The predicted stress level and the transformation slope $(\Sigma, T)$ are in good agreement with experiment. We can so conclude that our model is able to be adopted for the simulation of actuators exhibiting proportional and non-proportional (in the stress-temperature space) loadings.
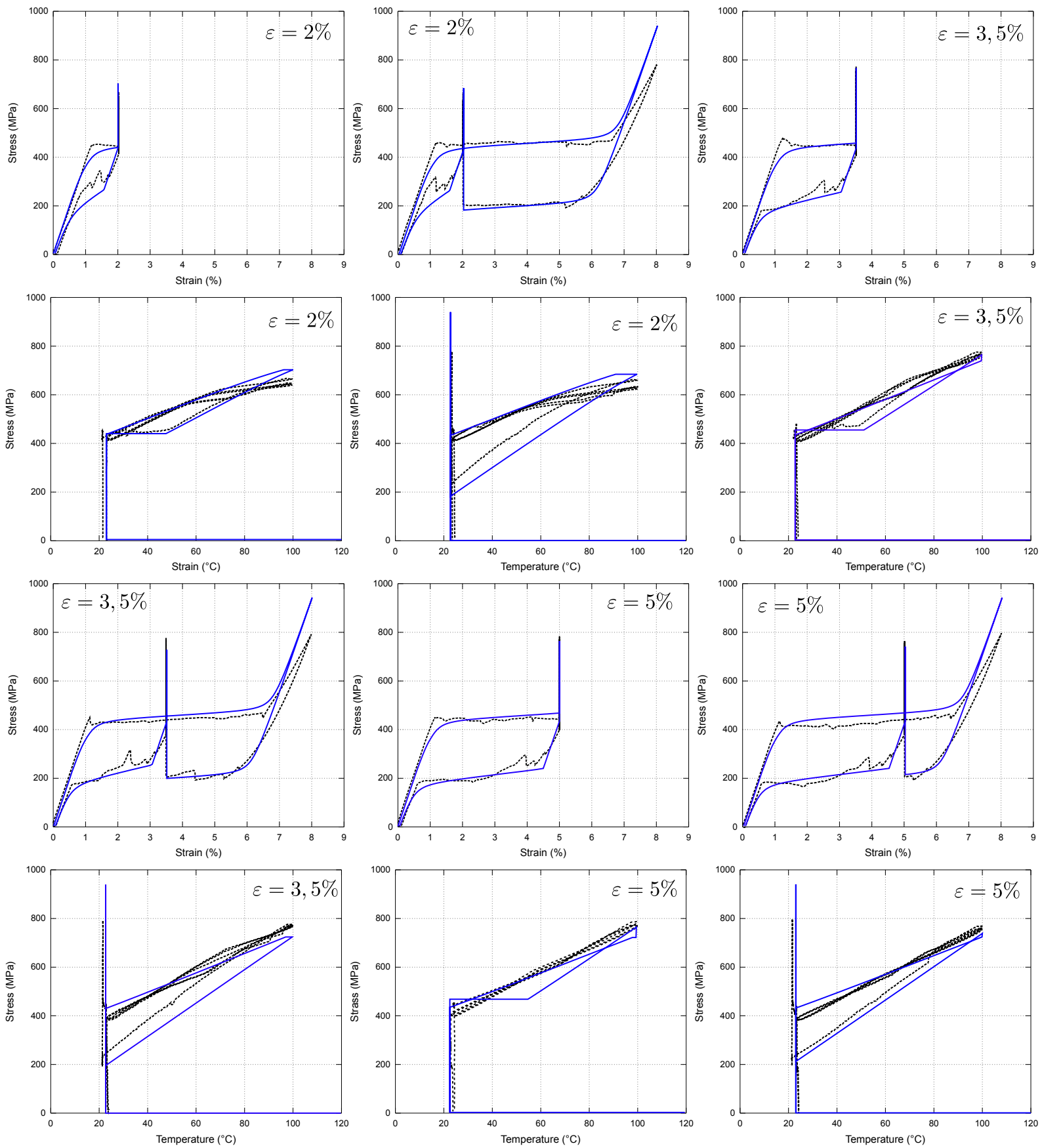

-..... Experimental - Simulation

Fig. 6. Stress recovery tests. 


\section{Conclusion}

The present model was designed in order to perform SMA structures simulations using FEA. In this RoundRobin, all model parameters were calibrated from uniaxial tension set unsing a reduced number of experiments. Comparisons between experiment and model predictions show a general good agreement. The uniaxial, actuation and stress-recovery paths show a very good predictive capability of the model based on this straightforward calibration. Only behaviors related to the occurence of a R-phase transformation are not captured. Some features of thetensiontorsion tests are correctly described. Several discrepancies in this last experiment set show the limits of the proposed model. Indeed, some additional characteristics like strain localization and material texture are not captured. The internal loops and the orientation/transformation couplings formulations extend the model capabilities to represent the material behavior for partial cycles and complete cycles in the vicinity of transformation temperatures. According to the discussion and observations made about the model capabilities, further work on SMA modeling will focus on taking into account the effect of material texture and the localization of transformation strain in small-size samples.

\section{References}

1. B. Peultier, T. Ben Zineb and E. Patoor, Mat. Science \& Eng. A, 481-482, (2008) 384-388

2. Y. Chemisky, PhD Thesis - Université de Metz (2009)

3. A. Duval, PhD Thesis - Nancy Université (2009)

4. C. Lexcellent and S. Leclercq, J. Mech. and Phys. of Solids, 44 (1996), 953-980

5. B. Wack, J.M. Terriez and P. Guelin, Acta Mechanica, 50 (1983), 9-37

6. Y.Chemisky, A. Duval, B. Piotrowski, T. Ben Zineb, V. Tahiri and E. Patoor, Smart Materials and Structures, 18 (2009) 104012 (10pp) 